\title{
Carotid cavernous fistula due to a ruptured intracavernous aneurysm of the internal carotid artery: treatment with selective endovascular occlusion of the aneurysm
}

\author{
I Wanke, A Doerfler, D Stolke, M Forsting
}

\begin{abstract}
Intracavernous carotid artery aneurysms causing a carotid-cavernous fistula (CCF) are rare. These aneurysms usually cause neurological symptoms due to gradual expansion without rupture. If they do rupture they most often lead to a CCF instead of bleeding into the subarachnoid space. A ptient is described with a ruptured intracavernous aneurysm causing a CCF resulting in acute onset of unilateral ophthalmoplegia. Selective coil embolisation of the aneurysm led to complete occlusion of the CCF with preservation of the internal carotid artery; symptoms resolved completely.

(F Neurol Neurosurg Psychiatry 2001;71:784-787)
\end{abstract}

Keywords: CCF; endovascular embolisation; intracavernous aneurysm; ophthalmoplegia

Aneurysms of the intracavernous portion of the carotid artery causing a carotid-cavernous fistula (CCF) are not often described in the literature. If they do rupture they cause a CCF rather than bleeding into the subarachnoid space. Treatment of choice is the endovascular approach.

\section{Case report}

A 33 year old women developed complete ptosis of her left eye over a period of 24 hours. She noted only mild headache without sudden onset and no history of trauma. Cranial CT, MRI, and digital subtraction angiography (DSA) were performed in an outside hospital; DSA disclosed a CCF on the left side with drainage into the cavernous sinus and petrosal sinus on both sides. There was no drainage via the superior ophthalmic vein.

Medical history disclosed a thrombosis of superficial veins of her left lower arm 5 years ago. The specimen taken from the vessel at that time did not show any pathology. Other than this event there was no other known illness. The patient did not show any external stigmata for a connective tissue disorder such as possible overexpansion of the joints or the skin. Her family history was unremarkable, and no connective tissue disorders were known.

On admission the patient was alert and fully oriented. Physical examination showed third
Department of Neuroradiology, University Hospital of Essen, Hufelandstrasse 55, D-45122 Essen, Germany

I Wanke

A Doerfler

M Forsting

Department of Neurosurgery

D Stolke

Correspondence to: Dr I Wanke

isabel.wanke@uni-essen.de

Received 15 February 2001 and in revised form

11 June 2001

Accepted 7 August 2001
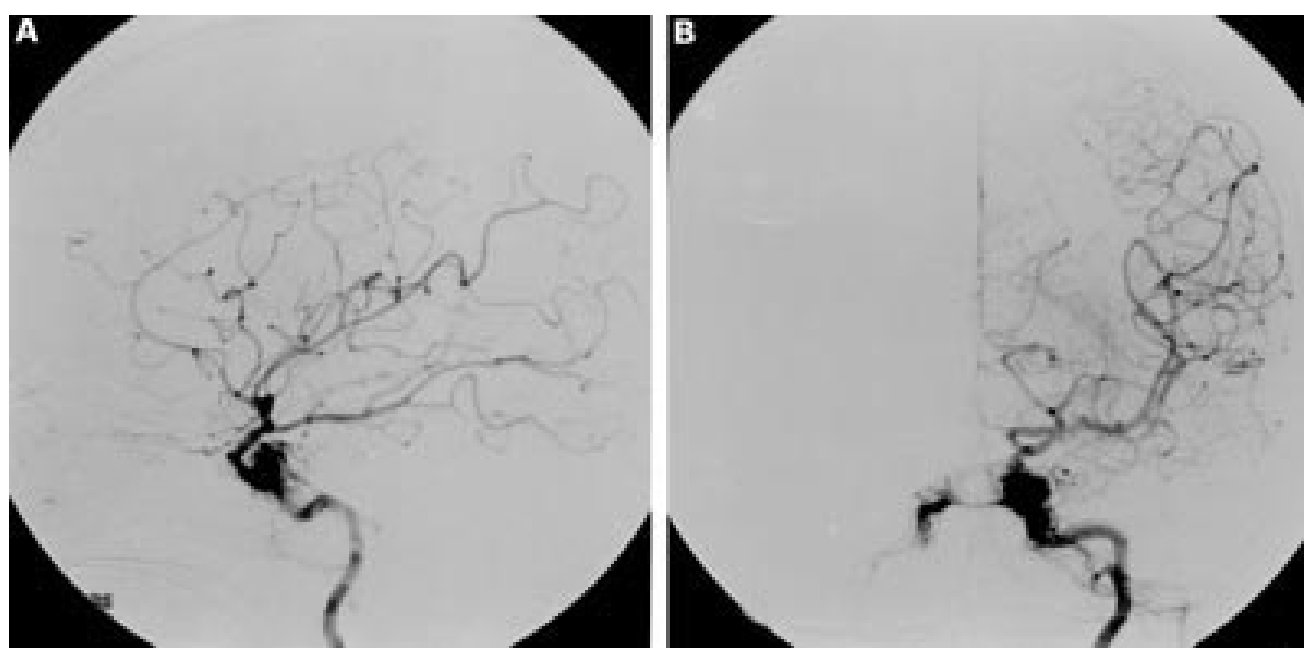

Figure 1 A left internal carotid artery angiogram (ICA); (A) lateral and $(B)$ anterioposterior view. These demonstrate a $C C F$ with direct shunting from the ICA into the cavernous sinus. The venous outflow from the fistula via the inferior petrosal sinus on the left side as well on the right side over the intercavernous sinus is well illustrated. The intracavernous aneurysm is not visible on these views. 


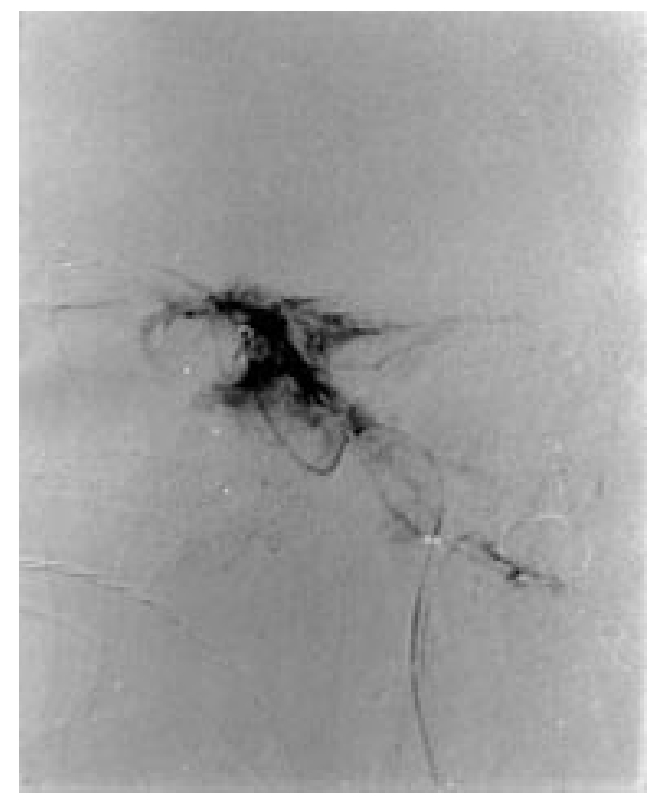

Figure 2 Lateral view through the microcatheter inserted through the fistula into the cavernous sinus.

and fourth cranial nerve palsy with complete left sided ptosis and lateral fixation of the bulbus, indicating that the sixth nerve was still working. There was neither paresis nor movement disturbances of the right eye. Ophthalmoscopy showed a normal papilla and fundus in each eye. The right pupil measured $3 \mathrm{~mm}$ and reacted normally to light whereas the left pupil was $5 \mathrm{~mm}$, did not react, and showed an afferent pupillary defect. Neither the patient nor the physician noted a cranial bruit. Routine blood count, serum electrolytes, urine analysis, prothrombin time, and platelet count were normal.

Selective cerebral angiography in our department showed a left CCF filled only from the left internal carotid artery, initially considered as a spontaneous CCF type A (fig $1 \mathrm{~A}$ and $\mathrm{B}$ ). As a variant there was a hypoplastic A1 segment on the left side with good collateralisation from the right side. Superselective angiography confirmed the initial diagnosis of a
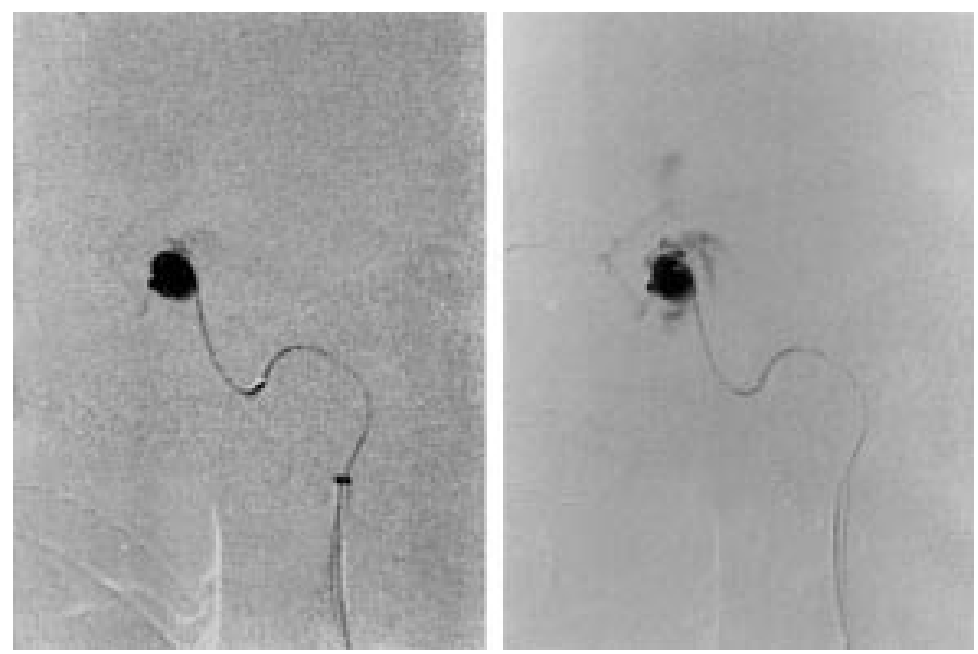

Figure 3 Aneurysmography through the retracted microcatheter positioned in the aneurysm lumen.
CCF (fig 2). Repositioning of the microcatheter (Excel 14, Target Therapeutics, Freemont, USA) with slight retraction of the tip towards the ICA vessel wall, however, showed a typical berry aneurysm with connection to the cavernous sinus (fig $3 \mathrm{~A}$ and $\mathrm{B}$ ). With the tip positioned within the aneurysm Guglielmi detachable coil (GDC) therapy was started. The aneurysm with an estimated size of $6 \mathrm{~mm}$ was occluded with two GDCs (GDC 10:6/20, GDC 10:4/8, Target Therapeutics, Freemont, USA). Control angiography showed obliteration of the aneurysm and total occlusion of the $\mathrm{CCF}$ (figure $4 \mathrm{~A}$ and $\mathrm{B}$ ).

We did not urge dense packing because we wanted to avoid a mass effect on the cavernous sinus. Three weeks later the patient had a control angiogram showing that the fistula and the aneurysm were still occluded. Clinically the patient improved dramatically and the ophthalmoplegia resolved.

\section{Discussion}

Several classifications of CCF exist depending on anatomy, aetiology, and pathophysiology. One categorisation divides between traumatic and spontaneous fistulas. Another classification is established according to fistulous supply to the cavernous sinus as follows: type A, internal carotid artery (ICA); type B, dural branches of the ICA; type C, dural branches of the external carotid artery (ECA); type D, combined forms. ${ }^{12}$ This leads to a further classification into direct, high flow, and indirect low flow fistulas because therapeutic management of CCF is strongly dependent on their haemodynamics. ${ }^{3-5}$ Finally, considering aetiology, there are several entities responsible for the development of a CCF: closed or penetrating head trauma, surgical damage, rupture of an intracavernous aneurysm, connective tissue disorders, vascular disease, and dural fistulas. ${ }^{67}$

Direct shunting CCFs are mainly caused by a tear of the internal carotid artery itself. In most cases, these CCFs are high flow, and spontaneous closure is rare as opposed to indirect shunting CCF with meningeal branches of the adjacent extracranial and intracranial arteries as feeding arteries. In these types spontaneous closure of the fistula can be expected in some cases. However, the clinical presentation of the patient is the most important factor. Hence, if the patient has visual impairment or cranial nerve dysfunction, treatment should be done as soon as possible to decompress the compromised nerve. This way irreversible damage can be avoided.

The sudden onset of clinical symptoms in our patient can be explained by the rupture of the aneurysm and sudden rise of blood pressure within the cavernous sinus. The third, fourth, and sixth cranial nerves are embedded in the lateral wall of the cavernous sinus with the sixth cranial nerve more medial and close to the ICA vessel wall. Ophthalmoplegia is due to pressure and distension of the nerves within the dural wall or the trabeculous structures of the cavernous sinus. Signs of a typical arteriovenous fistula were not present as there was a lack of chemosis, no bruit, and no pulsation of the eye globe. The 
A
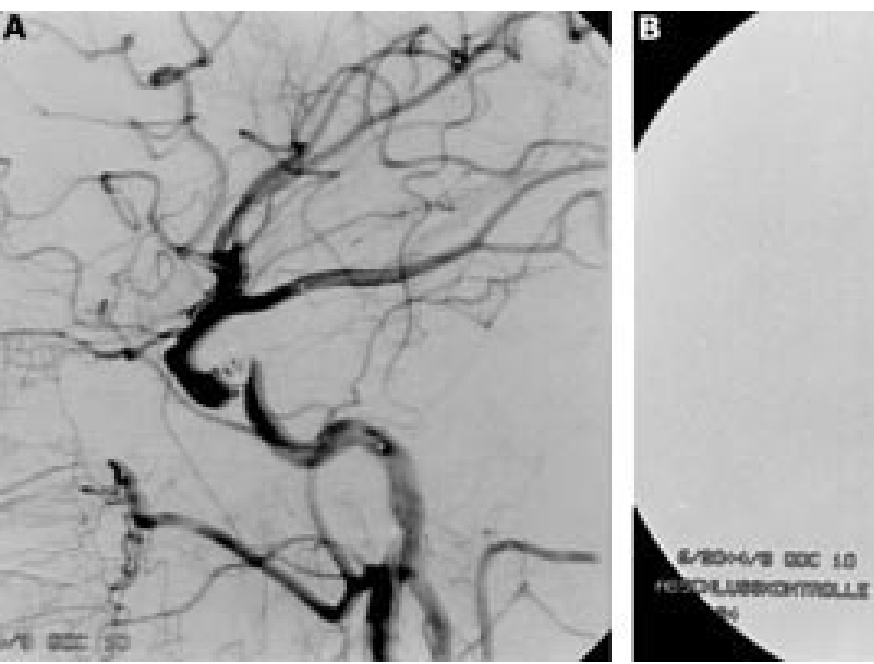

Figure 4 (A) A left lateral and (B) an anterioposterior view of the aneurym after insertion of GDCs with patency of the ICA.

superior ophthalmic vein was not dilated, which suggested an adequate outflow of the cavernous sinus into the petrosal sinus on both sides via the intercavernous sinus. In summary, symptomatology was more similar to a space occupying lesion of the cavernous sinus and not to an arteriovenous fistula.

The current treatment of choice of a CCF is an endovascular approach. Proximal ligation of the ICA by a surgical approach has been shown to be ineffective; in addition it prevents endovascular access to treat the fistula and may induce a severe steal phenomenon. ${ }^{8}$ Transarterial balloon occlusion of the fistula may be feasable in cases with direct shunting of the ICA into the cavernous sinus. ${ }^{910}$ In these cases the balloon is positioned through the arterial tear in the cavernous sinus adjacent to the ICA. After appropriate inflation of the balloon the fistula will usually be closed. In patients with a large fistulous connection it might become necessary to inflate a second or third balloon. Sometimes the cavernous portion of the ICA can be compressed or occluded by this manoeuvre. ${ }^{11}$ In addition, worsening or new onset of ophthalmoplegia after inflation of a detachable balloon in the cavernous sinus is possible. In patients with pre-existing occular symptoms it seems necessary to minimise the material delivered into the cavernous sinus to decrease the pressure on the compressed cranial nerves.

The development and experience with GDCs in the management of intracranial aneurysms led to the application of these in arteriovenous fistulas as well. ${ }^{12}$ We demonstrated a case of CCF caused by a ruptured pre-existing intracavernous aneurysm. It was classified as a spontaneous, direct fistula with direct supply from the ICA. The fistulous connection had to be expected to come from the aneurysm itself or from the carotid artery at the base of the aneurysm. The lack of reflux into the ophthalmic vein is unusual but explains why the patient did not experience typical symptoms of a CCF-like proptosis and chemosis. Because the lack of reflux into the ophthalmic vein is rare the possibility of an underlying aneurysm obstructing the anterior part of the cavernous sinus and thus the exit into the ophthalmic vein should be considered. Probably because of the high shunting blood volume, injection into the internal carotid artery did not show the aneurysm. There are some reported cases of intracavernous aneurysms causing CCF in the literature. ${ }^{6}{ }^{13}$ In our patient only superselective angiography showed a berry-like structure adjacent to the intracavernous carotid artery and bleeding was suggested from the ruptured dome of the aneurysm. Therefore closure of the fistula was carried out by selective occlusion of the aneurysm with detachable coils with preservation of the internal carotid artery. After intervention the patient's symptoms resolved. We could not find a reference reporting transarterial GDC embolisation of a ruptured aneurysm associated with a CCF, although two cases of GDC treatment via the venous route have been described. ${ }^{13} 14$

In some cases of CCF, we think that superselective angiography might be very helpful in demonstrating the aetiology of a high flow CCF. In cases of an underlying intracavernous aneurysm treatment could be focused only on the aneurysm itself. Therefore the mass effect on the cavernous sinus can be reduced because in most cases it will not be necessary to insert the coils into the sinus itself. In addition, the number of coils needed are less and therefore the treatment will be more cost effective.

\section{Conclusion}

In some cases, CCF may be caused by a ruptured intracavernous aneurysm with direct shunting into the cavernous sinus. To confirm the diagnosis of an underlying aneurysm it might be necessary to perform superselective angiography as in our patient. In such patients we think that endovascular GDC embolisation of the aneurysm with preservation of the parent artery should be considered as a therapeutic option.

1 Barrow DL, Spector RH, Braun IF, et al. Classification and treatment of spontaneous carotid-cavernous fistulas. $\mathcal{F}$ Neurosurg 1985;62:248-56. 
2 Peeters FLM, Kroger R. Dural and direct cavernous sinus fistulas. AfR Am f Roentgenol 1979;132:599-606.

3 Debrun GM. Endovascular management of carotid cavernous fistulas. In: Valavanis A. Interventional neuroradiology. Berlin, Germany: Springer, 1993:23-34.

4 Desal H, Leaute F, Auffray-Calvier E, et al. A fistule carotido-caverneuse directe: etudes clinique, radiologique et therapeutique. F Neuroradiol 1997;24:141-54

5 Vinuela F, Fox A, Debrun G, et al. Spontaneous carotid-cavernous fistulas: clinical, radiological, and therapeutic considerations. $\mathcal{F}$ Neurosurg 1984;60:976-84.

6 Hirai T, Korogi Y, Goto K, et al. Carotid-cavernous sinus fistula and aneurysmal rupture associated with fibromuscular dysplasia. Acta Radiol 1996;37:49-51.

7 Taki W, Nakhara I, Nishi S, et al. Pathogenetic and therapeutic considerations of carotid-cavernous sinus fistulas: Acta Neurochir (Wien) 1994;127:6-14.

8 Voris MC. Complications of ligation of the internal carotid artery. F Neurosurg 1980;53:22-7.
9 Larsen D, Higashida RT, Connors JJ. Treatment of carotidcavernous sinus fistulae. In: Interventional Neuroradiology. $215-26$.

10 Taki W, Handa H, Miyake H, et al. New detachable balloon technique for traumatic carotid cavernous sinus fistulae. AFNR Am f Neuroradiol 1985;6:961-4

11 Scialfa G. Vaghi A, Valsecchi F, et al. Neuroradiological treatment of carotid and vertebral fistulas and intracavernous aneurysms. Neuroradiology 1982;24:13-25.

12 Kahara Veikko J, Seppanen S, Kuurne T, et al. Endovascular reatment of carotid-cavernous fistulae. Acta Neurol Scand 998;98:254-8

13 Guglielmi G, Vinuela F, Briganti F, et al. Carotid-cavernous fistula caused by a ruptured intracavernous aneurysma: endovascular treatment by electrothrombosis with detachable coils. Neurorsurgery 1992;31:591-6.

14 Morris PP. Balloon reconstructive technique for the treatment of a carotid cavernous fistula. AfNR Am f Neutreatment of a carotid ca 